\title{
The epigenetic landscape of myalgic encephalomyelitis/chronic fatigue syndrome: deciphering complex phenotypes
}

\author{
Wilfred C de Vega ${ }^{1,2}$ \& Patrick O McGowan*,1,2,3,4 \\ ${ }^{1}$ Department of Biological Sciences, University of Toronto, Scarborough, ON, Canada \\ ${ }^{2}$ Department of Cell \& Systems Biology, University of Toronto, Toronto, ON, Canada \\ ${ }^{3}$ Department of Psychology, University of Toronto, Toronto, ON, Canada \\ ${ }^{4}$ Department of Physiology, Faculty of Medicine, University of Toronto, Toronto, ON, Canada \\ * Author for correspondence: patrick.mcgowan@utoronto.ca
}

"Our preliminary examinations of DNA methylation in ME/CFS have provided additional evidence for a major immune component and distinct profiles among groups of patients."

First draft submitted: 28 August 2017; Accepted for publication: 1 September 2017; Published online: 18 October 2017

Keywords: chronic fatigue syndrome $\bullet$ complex disease $\bullet$ disease subtyping $\bullet$ DNA methylation $\bullet$ epigenetics $\bullet$ glucocorticoid $\bullet$ HPA axis $\bullet$ immune system • myalgic encephalomyelitis

By their very nature, complex disease phenotypes are characterized by the dysregulation of multiple physiological systems, polygenic origins and various environmental triggers that result in patient populations with heterogeneous symptom profiles. Less than $10 \%$ of the heritability of complex phenotypes and disease traits are due to genetic variation, indicating that other factors play major roles in disease onset and progression [1]. Epigenetic modifications may partly account for this 'missing heritability' [2] through mechanisms that regulate transcriptional potential. These mechanisms appear to be, at least to some extent, responsive to environmental exposures or treatments. An improved understanding of the pathophysiology underlying complex phenotypes and new diagnostic tools can help refine and update classification criteria reliant on nonspecific or self-reported symptoms. Consequently, unraveling complex phenotypes depends to a large extent upon an ability to discriminate what are likely many distinct conditions. We and others have argued that epigenetic investigations integrate multiple levels of information (genetic, stochastic and environmental) to enable a better understanding of the dimensions of illness underlying complex phenotypes $[2,3]$. Here, we turn to myalgic encephalomyelitis/chronic fatigue syndrome (ME/CFS) to illustrate progress and future directions in this regard.

\section{Myalgic encephalomyelitis/chronic fatigue syndrome}

ME/CFS is a prototypical chronic illness of unknown etiology. The average age of symptom onset is 29-35 years, and women represent $75 \%$ of the clinical population [4]. Population estimates for the incidence of ME/CFS vary from 0.76 to $3.48 \%$ [5]. Most patients require tertiary care, resulting in an approximately $\$ 14$ billion annual cost to the US healthcare system due to the various co-morbid conditions and symptom profiles that necessitate multiple clinical visits and treatments [6].

$\mathrm{ME} / \mathrm{CFS}$ is currently defined by nonspecific symptoms of fatigue, pain, cognitive impairment and unrefreshing sleep, and then diagnosed by excluding medical and psychiatric diseases that explain the patient's symptoms [7]. This diagnosis identifies a highly heterogeneous patient population in terms of symptom profiles, which has been a barrier for researchers to understand the pathophysiology of ME/CFS [8]. However, a number of studies have reported immune and hypothalamic-pituitary-adrenal (HPA) dysfunction in ME/CFS patients.

\section{Biological differences observed in myalgic encephalomyelitis/chronic fatigue syndrome}

Studies that have investigated the immune response in ME/CFS patients have reported deficiencies in major cell types of the innate immune system, including natural killer cells exhibiting decreased cytotoxicity, cytotoxic $\mathrm{T}$ 
cells with reduced receptor expression and attenuated B cell response compared with healthy controls [9]. It has been difficult to determine the specific immune difference that may serve as the best clinical indicator of ME/CFS pathophysiology and whether these differences have a causative role. Nevertheless, immune deficiencies appear to be key features of ME/CFS.

The HPA axis, which exhibits extensive cross-talk with the immune system, regulates the endocrine response to stress and is hyporesponsive in ME/CFS. A number of studies have reported weakened diurnal variation of cortisol, mild hypocortisolism and a significantly stronger response to cortisol in patients [10]. One of the major mechanisms through which HPA regulation of the stress response occurs is by negative feedback inhibition activated by the binding of glucocorticoids (GCs) such as cortisol to GR in the brain and in the periphery. During a stress response, this binding inhibits endocrine release and leads to an upregulation of anti-inflammatory genes through the modification of cell signaling cascades. Considering that there are long-term effects on immune and HPA axis signaling in ME/CFS that do not appear to be explained by mutations of GR [11] or altered GR function [10], we examined epigenetic modifications in peripheral blood associated with ME/CFS.

\section{DNA methylation modifications in myalgic encephalomyelitis/chronic fatigue syndrome}

Research by our group using DNA microarrays identified methylation differences $>20 \%$ in 1192 CpG sites, corresponding to 826 genes, in peripheral blood mononuclear cells of ME/CFS patients [12]. Interestingly, a significant increase in hypomethylation was observed in promoter and regulatory regions of immune genes compared with other gene classes. We next explored the interaction of differential DNA methylation with downstream immune response to environmental stress by specifically examining GC signaling. An in vitro examination of peripheral blood mononuclear cell GC sensitivity using a T cell mitogen revealed two ME/CFS subtypes: a GC-hypersensitive subtype that included patients with enhanced negative feedback to GCs, and a GC-typical subtype showing sensitivity similar to controls [13]. Using statistical methods, GC sensitivity was isolated to methylation differences in 13 sites, mostly in noncoding regions but also in GST genes. By comparing both ME/CFS GC-sensitivity subtypes to healthy controls, we subsequently identified differential methylation in 4699 sites corresponding largely to cellular signaling and metabolic regulation as potential biomarkers for ME/CFS diagnosis. These functional differences in GC sensitivity could be mediated by DNA methylation modifications in specific T cell subsets, which have been observed in CD4 $T$ cells [14], or the alteration of alternative splicing patterns of GR, possibly through changes in the DNA methylation patterns of the various alternative exon promoters in the gene encoding GR $(N R 3 C 1)$ [15].

\section{Deciphering myalgic encephalomyelitis/chronic fatigue syndrome subtypes}

The potential utility of subtyping in ME/CFS in a manner that integrates phenotypic and biological data has been recognized for some time (e.g., [8]). ME/CFS immune subtypes have been described based on gene expression differences [9], dichotomous T cell GC sensitivity [13] and the varying efficacy of B cell therapy in relation to treatment [9]. Our preliminary examinations of DNA methylation in ME/CFS have provided additional evidence for a major immune component and distinct profiles among groups of patients. Together, these observations suggest that subtyping based on immune profiles may be useful in understanding the heterogeneity of ME/CFS and the relationship between these biological differences and symptom profiles $[7,9,10]$.

A challenge involved in combining multiple levels of data in clinical subtyping concerns the various scales and substructures of each data type. Previous work has attempted to identify ME/CFS subtypes through the concatenation of biological and clinical data, after normalizing these measures using principal component and latent class analyses. Using these methods, ME/CFS subtypes were assigned based on differences in sleep quality, obesity, menopausal status and cortisol levels [16]. However, simple concatenation can produce incoherent subtypes, where intrasubtype patient similarity is low, because common and complementary signals that can be used to produce stronger subtypes are removed or diluted $[17,18]$. Machine learning methods such as similarity network fusion (SNF) [18] and iCluster [17] have been created to address these challenges by retaining the unique substructures of each data type, resulting in subtypes that are more reflective of the full variability of the dataset. The selection of the appropriate method depends on the amount of data that is available for subtyping. iCluster appears to identify more coherent subtypes, according to silhouette scores - a statistical metric used to compare the efficiency of clustering - with a small number of preselected measurements, such as known biomarkers [17]. SNF integrates larger amounts of data to identify subtypes through iterative fusions of similarity matrices [18]. Using five different cancer datasets, SNF outperformed simple concatenation and iCluster by producing subtypes with higher silhouette scores, 
demonstrating its utility with larger datasets [18]. Machine learning approaches will likely enable more impactful aggregation of clinical and biological data, including genetic, epigenetic, transcriptomic and proteomic data, in order to identify and validate subtypes.

\section{Conclusion}

Mechanistic research is needed to understand how epigenetic modifications affect functional changes in ME/CFS, and whether or not such adverse epigenetic changes can be reversed or treated. An important step in the right direction is to identify and validate biomarkers for objective diagnosis. Two main approaches are needed: deep phenotyping of the disease and co-morbid conditions, focusing more on patients than case/(healthy) control comparisons, and integration of phenotype and biological data that includes epigenetic profiles. While detailed investigation of epigenetic marks other than DNA methylation modifications has yet to be performed in ME/CFS, increased HDAC expression [19] and an upregulation of micro RNA related to cell cycle and immune regulation [20] have been described, suggesting that these modifications may align with the DNA methylation results. Examining histone and RNA-based modifications in ME/CFS is required for a better understanding of the underlying disruptions to the overall epigenomic and gene expression dynamics in ME/CFS, and to determine if DNA methylation modifications in immune and HPA axis genes may serve as potential functional biomarkers for ME/CFS. In addition, subtyping analysis using biological information may be informative for other complex diseases that exhibit some of the prominent symptoms of ME/CFS such as postexertional malaise, which is observed in fibromyalgia [7]. Through the integration of published and publicly available biological and clinical data of diseases with similar clinical features, subsets that contain patients with different complex diseases will help identify the biological differences that are related to symptoms that are shared among patients. These comparisons will establish the biological differences that are unique to ME/CFS pathophysiology, assisting in refining diagnostic criteria and developing targeted therapeutics [8].

Financial \& competing interests disclosure

The authors have no relevant affiliations or financial involvement with any organization or entity with a financial interest in or financial conflict with the subject matter or materials discussed in the manuscript. This includes employment, consultancies, honoraria, stock ownership or options, expert testimony, grants or patents received or pending, or royalties.

No writing assistance was utilized in the production of this manuscript.

\section{References}

1. Frazer KA, Murray SS, Schork NJ, Topol EJ. Human genetic variation and its contribution to complex traits. Nat. Rev. Genet. 10(4), 241-251 (2009).

2. Petronis A. Epigenetics as a unifying principle in the aetiology of complex traits and diseases. Nature 465(7299), 721-727 (2010).

3. Sasaki A, De Vega WC, Mcgowan PO. Biological embedding in mental health: an epigenomic perspective. Biochem. Cell Biol. 91(1), 14-21 (2013).

4. Prins JB, Van Der Meer JW, Bleijenberg G. Chronic fatigue syndrome. Lancet 367(9507), 346-355 (2006).

5. Johnston S, Brenu EW, Staines D, Marshall-Gradisnik S. The prevalence of chronic fatigue syndrome/ myalgic encephalomyelitis: a meta-analysis. Clin. Epidemiol. 5, 105-110 (2013).

6. Lin JS, Resch SC, Brimmer DJ et al. The economic impact of chronic fatigue syndrome in Georgia: direct and indirect costs. Cost Eff. Resour. Alloc. 9(1), 1 (2011).

7. Fukuda K, Straus SE, Hickie I, Sharpe MC, Dobbins JG, Komaroff A. The chronic fatigue syndrome: a comprehensive approach to its definition and study. International Chronic Fatigue Syndrome Study Group. Ann. Intern. Med. 121(12), 953-959 (1994).

8. Jason LA, Corradi K, Torres-Harding S, Taylor RR, King C. Chronic fatigue syndrome: the need for subtypes. Neuropsychol. Rev. 15(1), 29-58 (2005).

9. Mensah FKF, Bansal AS, Ford B, Cambridge G. Chronic fatigue syndrome and the immune system: where are we now? Neurophysiol. Clin. 47(2), 131-138 (2017).

10. Papadopoulos AS, Cleare AJ. Hypothalamic-pituitary-adrenal axis dysfunction in chronic fatigue syndrome. Nat. Rev. Endocrinol. 8(1), 22-32 (2011).

11. Rajeevan MS, Smith AK, Dimulescu I et al. Glucocorticoid receptor polymorphisms and haplotypes associated with chronic fatigue syndrome. Genes Brain Behav. 6(2), 167-176 (2007).

12. De Vega WC, Vernon SD, Mcgowan PO. DNA methylation modifications associated with chronic fatigue syndrome. PLoS ONE 9(8), e104757 (2014). 


\section{Editorial de Vega \& McGowan}

13. De Vega WC, Herrera S, Vernon SD, Mcgowan PO. Epigenetic modifications and glucocorticoid sensitivity in myalgic encephalomyelitis/chronic fatigue syndrome (ME/CFS). BMC Med. Genomics 10(1), 11 (2017).

14. Brenu EW, Staines DR, Marshall-Gradisbik SM. Methylation profile of $\mathrm{CD}^{+} \mathrm{T}$ cells in chronic fatigue syndrome/myalgic encephalomyelitis. J. Clin. Cell. Immunol. 5, 228 (2014).

15. Vangeel E, Van Den Eede F, Hompes T et al. Chronic fatigue syndrome and DNA hypomethylation of the glucocorticoid receptor gene promoter 1F region: associations with HPA axis hypofunction and childhood trauma. Psychosom. Med. 77(8), 853-862 (2015).

16. Aslakson E, Vollmer-Conna U, Reeves WC, White PD. Replication of an empirical approach to delineate the heterogeneity of chronic unexplained fatigue. Popul. Health Metr. 7, 17 (2009).

17. Shen R, Olshen AB, Ladanyi M. Integrative clustering of multiple genomic data types using a joint latent variable model with application to breast and lung cancer subtype analysis. Bioinformatics 25(22), 2906-2912 (2009).

18. Wang B, Mezlini AM, Demir F et al. Similarity network fusion for aggregating data types on a genomic scale. Nat. Methods 11(3), 333-337 (2014).

19. Jason L, Sorenson M, Sebally K et al. Increased HDAC in association with decreased plasma cortisol in older adults with chronic fatigue syndrome. Brain Behav. Immun. 25(8), 1544-1547 (2011).

20. Brenu EW, Ashton KJ, Van Driel M et al. Cytotoxic lymphocyte microRNAs as prospective biomarkers for chronic fatigue syndrome/myalgic encephalomyelitis. J. Affect. Disord. 141(2-3), 261-269 (2012). 